\title{
Self-assembled nanofibrilar networks: Boosting hydrogelation efficiency by replacement of a pyridine moiety by a quinoline one
}

\author{
César A. Angulo-Pachón, Santiago Díaz-Oltra, , Juan J. Ojeda-Flores, Eva Falomir, Francisco Galindo, \\ and Juan F. Miravet*
}

Dedication ((optional))

\begin{abstract}
A new molecular hydrogelator consisting in a L-Valine derivative with appended quinoline units behaves as a supramolecular superhydrogelator forming gels at such low concentration as $0.8 \mathrm{mM}(0.05 \% \mathrm{w} / \mathrm{w})$. On the other hand, an analogue compound containing a pyridine moiety is found to be a poor gelator, forming gels at $19 \mathrm{mM}$. The gels are $\mathrm{pH}$ sensitive because of the protonation of the heterocycle and show microcrystallinity. The rheological properties and biocompatibility are also reported.
\end{abstract}

Molecular gels, as opposed to polymeric ones, are constituted by low molecular weight compounds that originate solvent percolating, self-assembled nanofibrillar networks. In the last decades there has been an increasing volume of research devoted to these soft materials. ${ }^{[1-3]}$ In particular, a main source of interest falls on the preparation of hydrogels due to their application in biomedical issues. ${ }^{[4]}$

Studies that rationalize molecular gel formation provide ground for the preparation of materials with the desired properties. Insight into the molecular details of self-assembled fiber formation have been reported recently. ${ }^{[5]}$ In the case of gels in organic solvents, solubility has been used to understand gelation efficiency. ${ }^{[6-9]}$ Much less studies have been carried out in this direction regarding gel formation in water although hydrophobic character ${ }^{[10]}$ and computational methods ${ }^{[11-12]}$ have been used to rationalize/predict gelation. Given a molecule capable of forming gels, choosing the structural variations required to improve its gelation efficiency, namely, the minimum gelator concentration required to form a hydrogel (mgc), is particularly challenging. Here, we address the issue of gelation efficiency, showing a dramatic improvement of gelation capabilities by a simple structural change.

Compound PyrVal3 (Scheme 1), which has been extensively studied by us, ${ }^{[13-15]}$ is a versatile molecular gelator both in organic solvents and water. The thermodynamic study of PyrVal3 in aqueous media revealed an entropy driven aggregation process dominated by the hydrophobic effect. ${ }^{[15]}$ In this study an analogue of PyrVal3 with an extended aromatic unit derived from quinoline, QuiVal3, is studied in detail and hydrogelation efficiency compared to related compounds PyrVal8 ${ }^{[16]}$ and QuiVal8. The initial hypothesis was that increased hydrophobic character and

[a] Dr. C. A. Angulo-Pachón, Dr. S. Díaz-Oltra, J. J. Ojeda-Flores, Dr. E. Falomir, Prof. F. Galindo and Prof. J. F. Miravet Department of Inorganic and Organic Chemistry University Jaume I

Avda. Sos Baynat s/n, 12071 Castellón, Spain

E-mail:miravet@uji.es

Supporting information for this article is given via a link at the end of the document.((Please delete this text if not appropriate)) improved $\pi-\pi$ stacking capabilities would result in a more efficient gelation process.

Gels were prepared by addition of $0.9 \mathrm{~mL}$ of water to $100 \mu \mathrm{L}$ of the gelator dissolved in DMSO. As shown in Table 1, quinoline derivatives with trimethylene and octamethylene central units formed gels with a rather remarkable efficiency. For example, the replacement of the pyridine unit at PyrVal3 by a quinoline one in QuiVal3 results in a dramatic improvement of the hydrogelation capabilities, reducing the $\mathrm{mgc}$ value from $19 \mathrm{mM}$ to $0.8 \mathrm{mM}$. The term supergelator has been used for molecules forming gel at such low concentrations. ${ }^{[17-18]}$

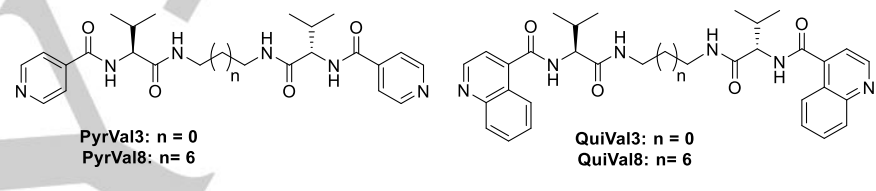

Scheme 1. Structure of the studied compounds.

Table 1. Minimum gelator concentration (mgc) of the studied compounds and their hydrophobic character measured with $\mathrm{Clog} \mathrm{P}$.

\begin{tabular}{cccc}
\hline Hydrogelator & $\mathrm{mgc} / \mathrm{mM}$ & $\mathrm{mgc} / \% \mathrm{w} / \mathrm{w}$ & $\mathrm{ClogP}$ \\
\hline PyrVal3 & 19 & 0.9 & 1.9 \\
PyrVal8 & 4 & 0.2 & 3.5 \\
QuiVal3 & 0.8 & 0.05 & 4.7 \\
QuiVal8 & 0.8 & 0.05 & 6.2 \\
\hline
\end{tabular}

A simple way to rationalize hydrogel formation efficiency is to consider the hydrophobic character of the compounds. For example, gel formation by a series of Fmoc-dipeptides has been related to $\log \mathrm{P}$ values (octanol-water partition coefficient values). ${ }^{[10]}$ Table 1 collects a comparison of mgc values of four related compounds and their hydrophobic nature measured as ClogP (octanol-water partition coefficient computed with the fragmental method implemented in ChemDraw). Introduction of an octamethylene spacer increases notably the hydrophobic character, changing the ClogP value from 1.9 for PyrVal3 to 3.5 for PyrVal8 and resulting in a ca. fivefold reduction of the mgc value. In accordance with this line of reasoning, QuiVal3 presents a higher $\mathrm{Clog} \mathrm{P}$ of 4.7 and much improved gelation efficiency, mgc $0.8 \mathrm{mM}$. Despite the fact that QuiVal8 presents much increased hydrophobicity, ClogP 6.2, gel formation is not improved compared to QuiVal3. The significant difference between

For internal use, please do not delete. Submitted_Manuscript 
pyridine and quinoline derived hydrogelators should be ascribed, aside of their different hydrophobic character, most likely to improved $\pi-\pi$ stacking of the extended aromatic units. Aromatic stacking is a main driving force for the aggregation in water and has been reported, for example, in the formation of hydrogels by peptides containing aromatic residues ${ }^{[19]}$ or amino acid or peptide derivatives containing the aromatic protecting group Fmoc. ${ }^{[20-21]}$ The identical mgc values of QuiVal3 and QuiVal8 despite their different hydrophobic character could be explained considering the entropic cost associated to the aggregation of such flexible species as QuiVal8.

A detailed study of QuiVal3 hydrogels was performed. Firstly, potentiometric titration revealed pKa values for the diprotonated and monoprotonated species respectively of 3.8 and 2.7 . Therefore below pH ca. 4 protonated species predominate which are water soluble and preclude gel formation.

As shown in Figure 1, analysis of xerogels obtained by lyophilization revealed an entangled fibrilar network composed of rather thin fibers with diameters below $50 \mathrm{~nm}$. X-ray diffraction of the xerogels indicates that the fibers are microcrystalline, presenting three mayor peaks at distances of 8.4, 5.2 and 4.6. (Figure 2). However, this observation is not a definitive proof of the crystallinity which may result from the drying process.
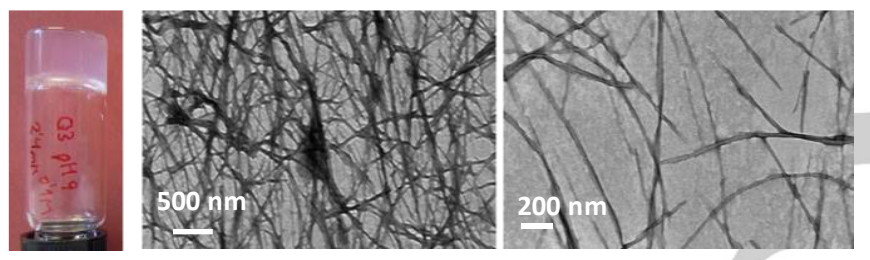

Figure 1. Transmission electron microscopy images of the xerogel formed by QuiVal3 and picture of the hydrogel.

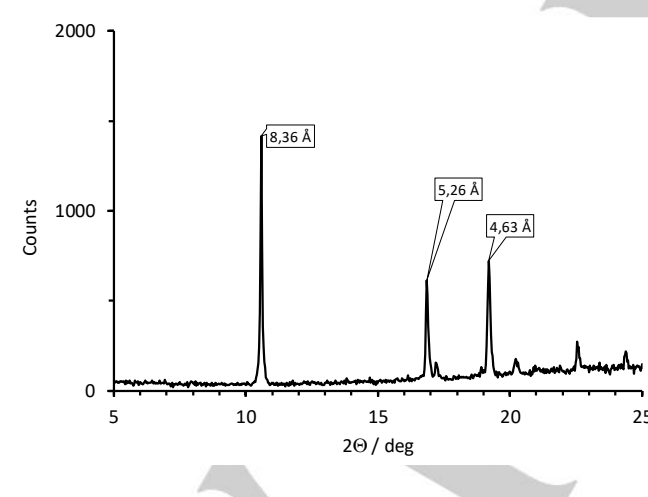

Figure 2. X-ray diffraction pattern of the xerogel formed by QuiVal3.

Additionally, rheological studies revealed the typical profile of gels, presenting values for the elastic modulus, G', well above those of the viscous modulus ( $\left.G^{\prime \prime}\right)$. The gel strength, as expected, is very dependent on the amount of fibrillar network and a plot of logG' vs log[QuiVal3] shows a linear correlation in agreement with the behavior reported for fibrillar thermoreversible gels formed by polymers $^{[22]}$ (Figure 3).
It is noteworthy the the hydrogel formed by QuiVal3 was able to incorporate the dye Rose Bengal within its fibrilar network, producing a rather intense increase in the molar absorptivity of the dye at $575 \mathrm{~nm}$. This fact could be be used to asses the critical concentration point associated to the aggregation onset of QuiVal3, which was found to be ca. $0.3 \mathrm{mM}$ (Figure 4).

Finally, envisaging future application of these hydrogels in biomedical areas, biocompatibility of QuiVal3 was assayed against healthy embrionary cells HEK-293, revealing that the molecules are biocompatible with LD50 values higher than 0.1 $\mathrm{mM}$ (see further details at $\mathrm{SI}$ ).

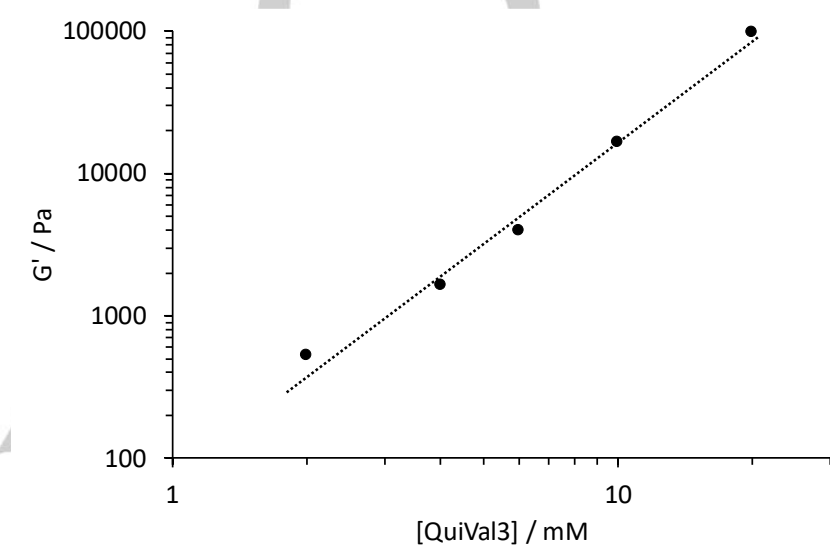

Figure 3. Dependence of rheological elastic modulus G' on the concentration of QuiVal3. Both axis present a logarithmic scale.

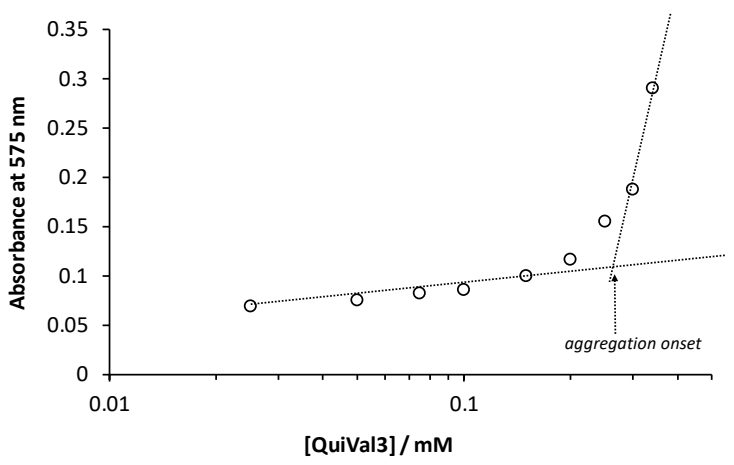

Figure 4. Variation of absorbance of Rose Bengal $(10 \mu \mathrm{M})$ with the concentration of QuiVal3

In summary the results presented highlight how the introduction of an extended aromatic unit such a quinoline boosts the hydrogelation affording a superhydrogelator. The comparison of the gelation capabilities of the four compounds presented in Table 1 suggest that although replacement of a quinoline unit by a pyridine one molecules increases the hydrophobic character, most likely the rather favorable $\pi-\pi$ stacking interactions provided by the quinoline heterocycle are a key factor in the observed behaviour. Overall, it has to be noted that the preparation of 
superhydrogelators is interesting for practical applications of molecular gels. On the one hand, reduced costs are associated to the minimum quantity of gelator required to form the gels. On the other hand, superhydrogelators produce gels that present much-reduced amounts of free, non-aggregated, gelator at equilibrium with the gel network.

\section{Experimental Section}

Synthesis of the compounds, NMR spectra, experimental procedures, additional rheological experiments and electron microscopy images can be found in the Supporting Information.

\section{Acknowledgements}

Ministerio de Economía y Competitividad of Spain (grant CTQ2015-71004-R) and Universitat Jaume I (grant P1.1B201576) are thanked for financial support. Technical support from SCIC of University Jaume I is acknowledged

.Keywords: supramolecular gels, molecular gels, hydrogels, selfassembled fibers

[1] J. W. Steed, Chem. Commun. 2011, 47, 1379-1383.

[2] R. G. Weiss, J. Am. Chem. Soc. 2014, 136, 7519-7530.

[3] E. R. Draper, D. J. Adams, Chem 2017, 3, 390-410.

[4] X. Du, J. Zhou, J. Shi, B. Xu, Chem. Rev. 2015, 115, 13165-13307
[5] C. D. Jones, S. R. Kennedy, M. Walker, D. S. Yufit, J. W. Steed, Chem 2017, 3, 603-628.

[6] J. Bonnet, G. Suissa, M. Raynal, L. Bouteiller, Soft Matter 2015, 11 2308-2312.

[7] K. K. Diehn, H. Oh, R. Hashemipour, R. G. Weiss, S. R. Raghavan, Soft Matter 2014, 10, 2632-2640.

[8] Y. Lan, M. G. Corradini, R. G. Weiss, S. R. Raghavan, M. A. Rogers, Chem. Soc. Rev. 2015, 44, 6035-6058.

[9] A. R. Hirst, I. A. Coates, T. R. Boucheteau, J. F. Miravet, B. Escuder, V. Castelletto, I. W. Hamley, D. K. Smith, J. Am. Chem. Soc. 2008, 130 9113-9121.

[10] D. J. Adams, L. M. Mullen, M. Berta, L. Chen, W. J. Frith, Soft Matter 2010, 6, 1971-1980.

[11] P. W. J. M. Frederix, G. G. Scott, Y. M. Abul-Haija, D. Kalafatovic, C. G. Pappas, N. Javid, N. T. Hunt, R. V. Ulijn, T. Tuttle, Nat. Chem. 2014, 7, 30.

[12] J. K. Gupta, D. J. Adams, N. G. Berry, Chem. Sci. 2016, 7, 4713-4719.

[13] J. A. Saez, B. Escuder, J. F. Miravet, Chem. Commun. 2010, 46, 79967998.

[14] J. F. Miravet, B. Escuder, Chem. Commun. 2005, 5796-5798.

[15] V. J. Nebot, J. Armengol, J. Smets, S. F. Prieto, B. Escuder, J. F. Miravet, Chem. Eur. J. 2012, 18, 4063-4072.

[16] V. J. Nebot, S. Díaz-Oltra, J. Smets, S. Fernándezprieto, J. F. Miravet, B. Escuder, Chem. Eur. J. 2014, 20, 5762-5767.

[17] R. Luboradzki, O. Gronwald, A. Ikeda, S. Shinkai, Chem. Lett. 2000, 1148-1149.

[18] S. J. Wezenberg, C. M. Croisetu, M. C. A. Stuart, B. L. Feringa, Chem. Sci. 2016, 7, 4341-4346.

[19] I. Maity, T. K. Mukherjee, A. K. Das, New J. Chem. 2014, 38, 376-385.

[20] Y. Zhang, H. W. Gu, Z. M. Yang, B. Xu, J. Am. Chem. Soc. 2003, 125, 13680-13681.

[21] R. Orbach, L. Adler-Abramovich, S. Zigerson, I. Mironi-Harpaz, D. Seliktar, E. Gazit, Biomacromolecules 2009, 10, 2646-2651.

[22] J. M. Guenet, J. Rheol. 2000, 44, 947-960. 
Entry for the Table of Contents (Please choose one layout)

Layout 1:

\section{COMMUNICATION}

The efficiency of the formation of selfassembled nanofibrilar networks that originate hydrogels is dramatically enhanced by replacement of a pyridine unit by a quinoline one in the described L-valine derived gelators.

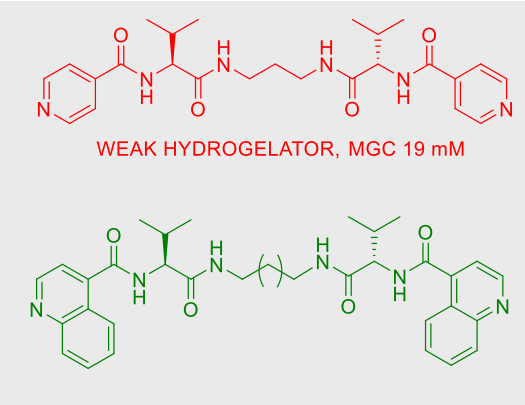

SUPERHYDROGELATOR, MGC $0.8 \mathrm{mM}$
César A. Angulo-Pachón, Santiago Díaz-Oltra, , Juan J. Ojeda-Flores, Eva Falomir, Francisco Galindo, and Juan F. Miravet*

Page No. - Page No.

Self-assembled nanofibrilar networks: Boosting hydrogelation efficiency by replacement of a pyridine moiety by a quinoline one

Layout 2:

\section{COMMUNICATION}

((Insert TOC Graphic here))
Author(s), Corresponding Author(s)*

Page No. - Page No.

Title

Text for Table of Contents 
Additional Author information for the electronic version of the article.

$\begin{array}{ll}\text { Author: } & \text { ORCID identifier } \\ \text { Author: } & \text { ORCID identifier }\end{array}$

Author: $\quad$ ORCID identifier 